\title{
Activity of $1-\left(2^{\prime}\right.$-Fluoro-2'-Deoxy- $\beta$-D- Arabinofuranosyl)Thymine Against Herpes Simplex Virus in Cell Cultures and Rabbit Eyes
}

MELVIN D. TROUSDALE, ${ }^{1 *}$ ANTHONY B. NESBURN,${ }^{1}$ TSANN-LONG SU ${ }^{2}$ CARLOS LOPEZ, ${ }^{2}$ KYOICHI A. WATANABE, ${ }^{2}$ AND JACK J. FOX ${ }^{2}$

Estelle Doheny Eye Foundation and Department of Ophthalmology, University of Southern California School of Medicine, Los Angeles, California $90033^{1}$ and Sloan-Kettering Institute for Cancer Research, Laboratories of Organic Chemistry and Herpesvirus Infections, Memorial Sloan-Kettering Cancer Center, Sloan-Kettering Institute, New York, New York $10021^{2}$

Received 9 December 1982/Accepted 22 March 1983

\begin{abstract}
A new antiviral compound 1-(2'-fluoro-2'-deoxy- $\beta$-D-arabinofuranosyl)thymine (2'-fluoro-5-methyl-ara-uracil [FMAU]), was compared with acyclovir and idoxuridine in vitro against two strains of both herpes simplex virus type 1 (HSV-1) and HSV-2. Determinations of the $50 \%$ effective dose varied slightly with each strain and with the host cells employed. The $50 \%$ effective dose for FMAU and acyclovir against HSV-1 ranged from $0.1 \mu \mathrm{M}$ to 0.5 to $0.6 \mu \mathrm{M}$ in rabbit kidney cells and from $0.5 \mu \mathrm{M}$ to 0.6 to $0.78 \mu \mathrm{M}$ in Vero cells. Beginning 4 days postinoculation, topical FMAU therapy given five times per day to rabbits with acute herpetic keratitis either suppressed or delayed the severity of corneal epithelial involvement, conjunctivitis, iritis, and corneal clouding. Responses to treatment with FMAU were similar to those obtained with acyclovir and significantly better than those attained with idoxuridine and vidarabine. At 30 to 40 days after the end of treatment, rabbit eyes were subjected to iontophoresis with epinephrine in an attempt to induce reactivation and enhance detection of previously latent HSV-1. Latent HSV-1 was detected in 67 to $92 \%$ of trigeminal ganglia in FMAU-treated animals and in $90 \%$ of placebo-treated animals.
\end{abstract}

Herpes simplex virus (HSV) infection of the eye is the most serious and most prevalent infectious cause of corneal blindness in the United States (15). Several antiviral compounds, including idoxuridine (IDU), vidarabine (adenine arabinoside [Ara-A]), trifluridine, and acyclovir (ACV), are available for use against ocular herpes infection (11) and are most helpful in the treatment of superficial herpetic keratitis. None of the currently available antiviral agents, however, can prevent the establishment of latent disease when delivered after the appearance of overt infection, prevent spontaneous recurrence, or eradicate latent $\mathrm{HSV}$ from the nervous system $(2,8,9)$. Therefore, the search continues for a means to prevent and control both acute and recurrent herpetic disease in humans.

A new family of nucleoside analogs has been synthesized, and studies indicate that the substitutions at $\mathrm{C}-5$ and $\mathrm{C}-2^{\prime}$ are important in determining biological activity $(10,20)$. Two of these compounds, 1 - $\left(2^{\prime}\right.$-deoxy-2'-fluoro- $\beta$-D-arabinofuranosyl)-5-iodocytosine ( $2^{\prime}$-fluoro-5-iodoara-C [FIAC]) and 1-( $2^{\prime}$-fluoro- $2^{\prime}$-deoxy- $\beta-D-$ arabinofuranosyl)thymine (2'-fluoro-5-methylara-uracil [FMAU]) (Fig. 1), were found to be both potent and selective against HSV type 1 (HSV-1) and HSV-2 by in vitro studies $(10,13)$. In vivo studies in mice inoculated with HSV-1 showed that both FIAC and FMAU were effective as antiviral agents (10). Topical FIAC therapy was found to be beneficial against acute ocular herpetic keratitis in rabbits (19). The specificity of FIAC and FMAU, like other promising new antiherpetic drugs, appears to be dependent, at least in part, upon an interaction with the virus-specified thymidine kinase $(7,10)$.

The studies presented here were designed to compare the in vitro antiherpetic activity of FMAU, ACV, and IDU against HSV-1 and HSV-2 in secondary rabbit kidney (SRK) and Vero cell cultures and to evaluate the topical antiviral effect of FMAU, ACV, IDU, and Ara$A$ on acute ocular keratitis in rabbits.

\section{MATERIALS AND METHODS}

Virus strains. Two different strains of HSV-1, the standard laboratory McKrae strain (our laboratory) and the RE6 strain (provided by Y. M. Centifanto, Louisiana State University Medical Center, New Orleans), were used in in vitro studies. Two HSV-2 strains, HG52 (provided by John Subak-Sharpe, Insti- 
<smiles>NC1=NC([O-])N(C23OC(CO)[C@@H](OC2C(F)(F)F)[C@H]3O)C=I1</smiles><smiles></smiles>

FIG. 1. Structures of FIAC and FMAU.

tute of Virology, Glasgow, Scotland) and 333 (provided by Richard Dix, Veterans Administration Medical Center, San Francisco, Calif.), were also evaluated in vitro. Each strain was propagated and quantitated on monolayers of SRK cell cultures.

Cell cultures. Growth medium for both SRK and Vero cell cultures consisted of minimum essential medium (GIBCO Laboratories, Grand Island, N.Y.) supplemented with $10 \%$ fetal calf serum, $20 \%$ NCTC135, $2 \mathrm{mM}$ L-glutamine, $10 \mathrm{mg}$ of gentamicin per $\mathrm{ml}$, and $3 \mathrm{mg}$ of amphotericin B per $\mathrm{ml}$ (Fungisone, E. R. Squibb \& Sons, Princeton, N.J.)

In vitro evaluation of antiviral activity. Confluent monolayers of SRK and Vero cells were inoculated with ca. 100 PFU of virus and allowed to adsorb for $1 \mathrm{~h}$ at room temperature. The virus inoculum was removed, and the cell monolayers were overlaid with $2 \%$ methylcellulose medium containing a threefold serial dilution of the antiviral drug (19). After incubation for $72 \mathrm{~h}$ at $37^{\circ} \mathrm{C}$ in a humidified $5 \% \mathrm{CO}_{2}$ atmosphere, the medium was discarded. Cells were fixed with methanol-acetic acid (3:1) and stained with $0.5 \%$ crystal violet. The number of plaques formed in the presence and absence of the drug was determined and plotted as a percentage of the control. The theoretical concentration of drug which would give a $50 \%$ plaque reduction (i.e., $50 \%$ effective dose) was then determined from the scale.

Infection and treatment of rabbit eyes. Both eyes of each of 30 New Zealand white male rabbits weighing 3 to $4 \mathrm{~kg}$ were inoculated without scarification. Each eye received $0.1 \mathrm{ml}$ of the McKrae strain of HSV-1 containing $10^{5}$ PFU. Virus inoculum was placed in the lower conjunctival cul de sac, and the lid was closed and gently rubbed for $30 \mathrm{~s}$, at which time the process was repeated. On day 3 post-inoculation, all eyes were stained with $0.5 \%$ methylene blue and examined by slit-lamp biomicroscopy. The rabbits were then divided into groups matched on the basis of herpetic corneal epithelial involvement.

To determine the optimum concentration of FMAU for topical use, 10-fold serial dilutions of this agent, ranging from 0.2 to $0.0002 \%$, were prepared in $0.85 \%$ saline. On day 4 after viral inoculation, all corneas were treated topically with 1 drop $(0.05 \mathrm{ml})$ of the appropriate drug dilution five times per day (8 a.m., 11 a.m., 2 p.m., 4 p.m., and 10 p.m.) for 11 consecutive days.

For a comparative study, other rabbits (five animals per treatment group) infected with HSV-1 (McKrae strain) under similar experimental conditions received coded drug treatments, which included ACV (3\% Zovirax ophthalmic ointment), Ara-A (3\% Vira-A ophthalmic ointment), IDU (0.5\% Stoxil ophthalmic ointment), and $0.85 \%$ saline as the placebo.

Beginning on day 4 a single examiner, uninformed with respect to drug regimen, examined all eyes daily for corneal epithelial involvement (epithelial keratitis), conjunctivitis, iritis, and corneal clouding. A grading system of 0 to $4+$ with one-quarter-step intervals below $1+$ and one-half-step intervals above $1+$ was used; $1+, 2+, 3+$, and $4+$ were equivalent to 25,50 , 75 , and $100 \%$ involvement of the corneal area examined.

Two-way analysis of variance with repeated measures was performed to test for differences in the mean degree of severity across the five treatment groups (21). Multiple comparisons, using the Student-Newman-Keuls procedure, were performed between pairs of treatment groups (21). The significance level alpha was set at 0.05 .

Induction of ocular HSV shedding by iontophoresis. At 30 to $\mathbf{4 0}$ days after the end of treatment, all surviving rabbits were stimulated to induce HSV ocular recurrence to enhance detection of virus from the nervous system. Stimulation was accomplished by iontophoresis of $0.01 \%$ epinephrine into the corneal epithelium with direct current $(0.8 \mathrm{~mA} ; 9 \mathrm{~V})$ for $8 \mathrm{~min}$ once a day for 3 consecutive days (12).

Isolation of HSV from tear films and tissue. After the initiation of iontophoresis, tear film samples were taken daily with cotton swabs and inoculated onto monolayers of SRK cells at $37^{\circ} \mathrm{C}$. Monolayers were examined microscopically for typical HSV cytopathic effects for 7 to 10 days.

On day 7 after iontophoresis, the rabbits were sacrificed and portions of both trigeminal ganglia, cortex, midbrain, and cerebellum were removed aseptically. To further enhance detection of latent HSV, the samples were then enzymatically disbursed as previously described (14). Enzymatically disbursed cells were cocultivated with SRK cell monolayers and examined for cytopathic effects every other day for 21 to 28 days.

\section{RESULTS}

In vitro antiherpetic activities of FMAU. The antiherpetic activities of FMAU, ACV, and IDU, as determined by the plaque reduction method, are presented in Table 1. FMAU and ACV had about the same activity against both strains of HSV-1 and HSV-2 when tested in either SRK or Vero cell cultures. The $50 \%$ effective dose of FMAU and ACV against HSV1 and HSV-2 was between 0.10 and $0.6 \mu \mathrm{M}$ in SRK cells and 0.09 and $0.78 \mu \mathrm{M}$ in Vero cells. IDU had less potent antiviral activity against the same strains of HSV-1 and HSV-2. The 50\% effective doses for IDU were between 0.7 and $1.7 \mu \mathrm{M}$ in SRK cells and 1.0 and $1.4 \mu \mathrm{M}$ in Vero cells.

Topical FMAU treatment of acute herpetic keratitis. Topical FMAU was well tolerated by rabbit eyes; no toxicity was demonstrated when normal eyes were treated five times per day for 2 
TABLE 1. Antiherpetic activity of FMAU, ACV, and IDU in SRK and Vero cells

\begin{tabular}{|c|c|c|c|c|c|c|}
\hline \multirow{3}{*}{ Virus } & \multicolumn{6}{|c|}{$50 \%$ Effective dose $(\mu \mathrm{M})$ in: } \\
\hline & \multicolumn{3}{|c|}{ SRK cells } & \multicolumn{3}{|c|}{ Vero cells } \\
\hline & FMAU & ACV & IDU & FMAU & ACV & $\overline{\text { IDU }}$ \\
\hline HSV-1 (McKrae) & 0.16 & 0.1 & 1.1 & 0.78 & 0.6 & $\overline{1.1}$ \\
\hline HSV-1 (RE6) & 0.61 & 0.5 & 0.7 & 0.51 & 0.5 & 1.0 \\
\hline HSV-2 (HG52) & 0.13 & 0.5 & 1.6 & 0.09 & 0.5 & 1.4 \\
\hline HSV-2 (333) & 0.52 & 0.5 & 1.7 & 0.40 & 0.6 & 1.3 \\
\hline
\end{tabular}

weeks with $0.2 \%$ FMAU drops (data not shown). No noticeable adverse reactions of any kind were observable in any of the animals.

Superficial punctate lesions and dendrites appeared by day 4 after inoculation, at which time the rabbits were separated into matched groups based on the severity of their ocular disease and the treatment initiated. In the placebo-treated eyes, large dendrites and geographic ulcers developed during days 5 to 8 (Fig. 2a). By day 9 post-inoculation, the severity of corneal epithelial involvement began to clear and, except for mild nonspecific punctate keratopathy, appeared clear by day 14 . The degrees of severity
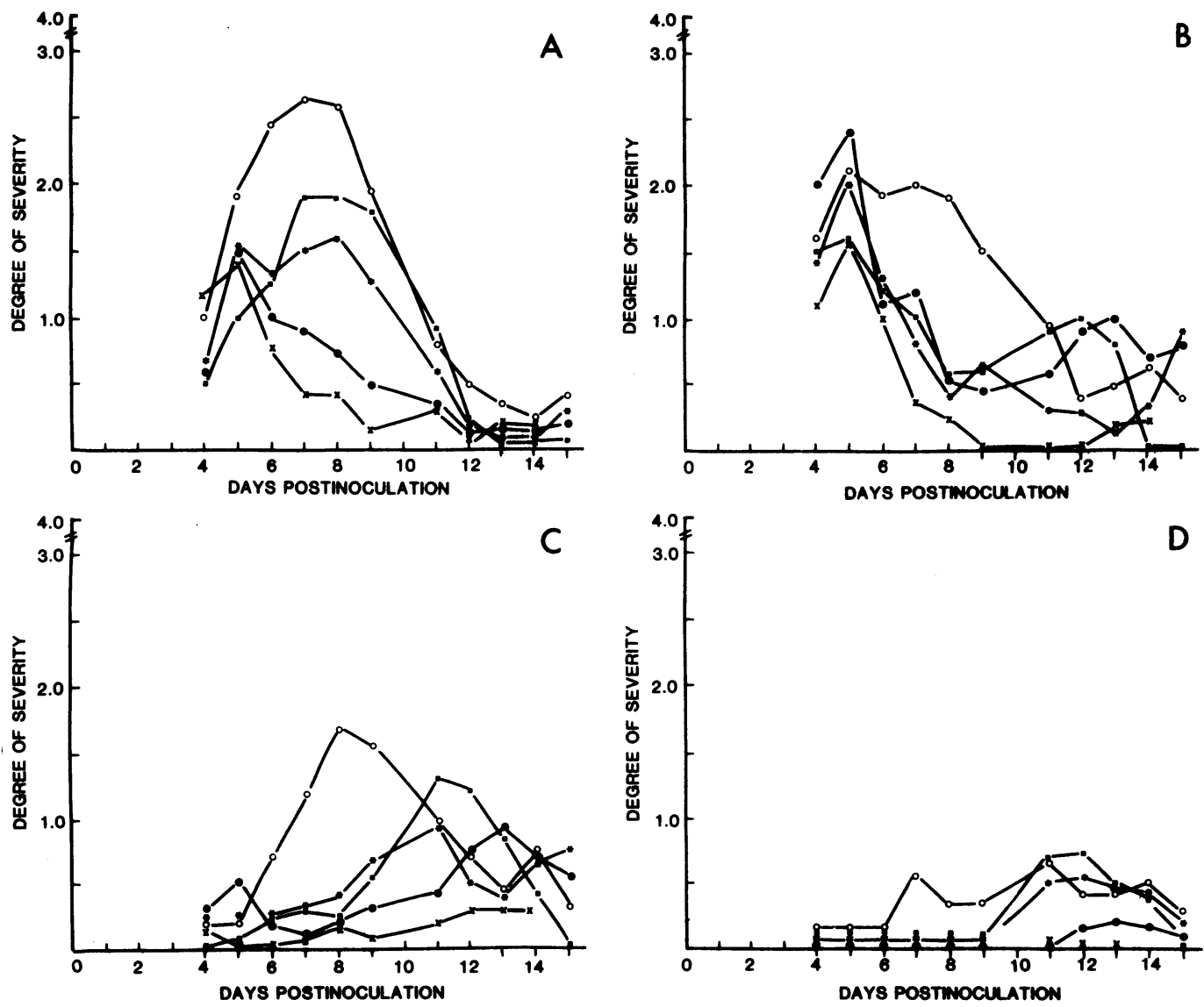

FIG. 2. Degree of severity of corneal epithelial involvement (A), conjunctivitis (B), iritis (C), and corneal clouding (D), observed in young adult New Zealand white male rabbits after inoculation with $10^{5}$ PFU of HSV-1 (McKrae). Corneas were not scarified. Drops were given topically five times per day for 11 consecutive days beginning on day 4 post-inoculation. Each treatment group consisted of six animals (12 eyes) receiving either 0.2 , $0.02,0.002$, or $0.0002 \%$ FMAU or $0.85 \%$ saline (placebo). Symbols: $\times, 0.2 \%$ FMAU; $0.02 \%$ FMAU; ${ }^{*}$, $0.002 \%$ FMAU; $\mathbf{0}, 0.0002 \%$ FMAU; and $O$, placebo (saline). 
TABLE 2. Isolation of HSV from FMAU-treated rabbits after iontophoresis of epinephrine to induce reactivation of latent $\mathrm{HSV}$

\begin{tabular}{lccccc}
\hline \multirow{4}{*}{$\begin{array}{c}\text { Treatment } \\
\text { group }\end{array}$} & $\begin{array}{c}\text { Trigeminal } \\
\text { ganglia }\end{array}$ & Cortex & $\begin{array}{c}\text { Mid- } \\
\text { brain }\end{array}$ & $\begin{array}{r}\text { Cere- } \\
\text { bellum }\end{array}$ \\
\cline { 2 - 6 } & Left & Right & & & \\
\hline $0.2 \%$ & $4 / 6$ & $4 / 6$ & $2 / 6$ & $1 / 6$ & $0 / 6$ \\
$0.02 \%$ & $5 / 6$ & $6 / 6$ & $5 / 6$ & $1 / 6$ & $2 / 6$ \\
$0.002 \%$ & $5 / 6$ & $5 / 6$ & $2 / 6$ & $2 / 6$ & $1 / 6$ \\
$0.0002 \%$ & $5 / 6$ & $5 / 6$ & $0 / 6$ & $1 / 6$ & $1 / 6$ \\
Placebo & $4 / 5$ & $5 / 5$ & $2 / 5$ & $1 / 5$ & $0 / 5$ \\
\hline
\end{tabular}

a Number of positive virus samples over the total samples tested.

of corneal epithelial involvement in treatment groups receiving 0.2 and $0.02 \%$ FMAU were similar to each other; in both of these groups maximum corneal epithelial involvement was reached by day 5 , after which involvement diminished. Eyes receiving either 0.002 or $0.0002 \%$ FMAU also gave similar responses, with maximum corneal epithelial involvement being recorded on day 7 or 8 at a level of severity between 1.5 and 1.8 . This was significantly less than the severity displayed in the placebo group.

The severity of conjunctivitis was reduced after 1 to 2 days of FMAU treatment regardless of drug concentration (Fig. 2b). This statistically significant $(P<0.01)$ reduction in conjunctivitis was evident from days 6 to 9 . From days 9 to 12, there was no evidence of conjunctivitis in eyes treated with $0.2 \%$ FMAU. On days 13 to 14 there was evidence of recurrence. At lower concentrations of FMAU (i.e., 0.02, 0.002, and $0.0002 \%$ ), there was a secondary increase in the severity of conjunctivitis after day 10 .

Compared with the placebo-treated eyes, minimal or no iritis (degree of severity, $<0.5$ ) was observed when FMAU chemotherapy was initiated (Fig. 2c). Iritis in placebo-treated eyes increased substantially from days 5 to 8 , reaching a degree of severity of ca. 1.7, and then declined for 5 consecutive days to a mean severity score of 0.4 . Both the 0.2 and $0.02 \%$ FMAU treatments suppressed the severity of iritis and delayed the time to peak levels until days 11 to 12. Treatment with the more dilute concentrations of FMAU resulted in less delay and depression of iritis.

Corneal clouding (Fig. 2d) was observed initially on day 4 in the placebo group ( 4 of 12 eyes positive) and increased significantly by day 7 ( 7 of 12 eyes positive). Corneal clouding was either suppressed or delayed in all drug-treated groups. Only two eyes (same animal) receiving $0.2 \%$ FMAU exhibited clouding during the study; 2 of 12 eyes treated with $0.02 \%$ FMAU displayed clouding, but not until day 12. Eyes treated with either 0.002 or $0.0002 \%$ FMAU developed clouding at about the same level of severity as the placebo group, but the response was delayed by several days.

After $24 \mathrm{~h}$ of FMAU treatment (five topical applications), HSV-1 was still isolated from tear film samples from 22 of 24 rabbits (data not shown). Regardless of the drug concentration employed, HSV-1 was able to infect the nervous system and establish a latent infection. For example, latent HSV-1 was readily demonstrated in FMAU-treated rabbits when the animals were stimulated to induce reactivation at 30 to 40 days after completion of chemotherapy (Table 2). A majority of the trigeminal ganglion samples from the FMAU-treated rabbits yielded latent HSV (8 of 12 to 11 of 12, or 67 to $92 \%$ ), as did samples from the placebo-treated rabbits $(9$ of 10 , or $90 \%$ ). HSV-1 was isolated less frequently from cortex, midbrain, and cerebral tissues.

In vivo antiherpetic activity of topical FMAU, ACV, IDU, and Ara-A. Groups of five to six rabbits with acute ocular herpetic keratitis were treated topically with either FMAU, ACV, IDU, Ara-A, or placebo. The day-to-day results of ocular examinations are presented in Table 3. Although several ocular parameters (corneal epithelial involvement, conjunctivitis, iritis, and corneal clouding) were tabulated on a daily

TABLE 3. Severity of corneal epithelial involvement during treatment of acute herpetic keratitis with FMAU, ACV, IDU, and Ara-A

\begin{tabular}{clllll}
\hline \multirow{2}{*}{$\begin{array}{c}\text { Day post- } \\
\text { inoculation }\end{array}$} & \multicolumn{5}{c}{ Severity $^{a}$ with following treatment: } \\
\cline { 2 - 6 } & FMAU & ACV & IDU & Ara-A & Placebo \\
\hline 4 & 1.19 & 1.28 & 1.30 & 1.95 & 2.25 \\
5 & 1.46 & 1.15 & 1.20 & 2.25 & 2.65 \\
6 & 0.77 & 0.95 & 2.30 & 2.85 & 3.60 \\
7 & 0.44 & 0.80 & 1.95 & 3.20 & 4.00 \\
8 & 0.44 & 0.60 & 2.00 & 2.35 & 3.75 \\
9 & 0.15 & 0.43 & 1.08 & 2.03 & 3.65 \\
10 & ND $^{b}$ & 0.38 & 1.25 & 1.60 & 2.75 \\
11 & 0.33 & 0.30 & 1.13 & 1.30 & 1.65 \\
12 & 0.38 & 0.25 & 0.95 & 1.33 & 1.13 \\
13 & 0.21 & 0.20 & 0.43 & 1.20 & 1.28 \\
& & & & & \\
Avg $^{c}$ & $0.58 *$ & $0.63^{*}$ & $1.36 \dagger$ & $2.01 \dagger \dagger$ & $2.67 \dagger \dagger \dagger$ \\
\hline
\end{tabular}

a Mean values were determined from 12 eyes in the FMAU treatment group and 10 eyes in the other groups. The grading system was on a 0 to $4+$ scale as described in the text.

$b$ ND, Not determined.

c Analysis of variance revealed significant differences in average severity across the five treatment groups $(P<0.05)$. Averages marked with the same symbol (e.g., * and $\dagger$ ) are not significantly different from one another as determined by the Student-Newman-Keuls procedure. 
basis, corneal epithelial involvement was chosen to demonstrate the effectiveness of the chemotherapeutic agents. Multiple comparisons, using the Student-Newman-Keuls procedure, revealed that the mean severity across treatments differed as follows: FMAU = ACV < IDU < Ara-A $<$ placebo (Table 3). Thus, on the average, the corneal epithelial involvement of rabbits receiving either FMAU or ACV therapy was significantly less than that of those receiving IDU, Ara-A, or placebo $(P<0.05)$. Eyes treated with IDU or Ara-A responded favorably; however, their response was slower and less dramatic. Corneal involvement in eyes treated with saline only was significantly greater on the average than that of the four drug-treatment groups.

\section{DISCUSSION}

Comparison of in vitro tests for the evaluation of relative potencies of antiviral drugs is difficult because test procedures vary among laboratories $(2,17,19)$. In recent independent studies, De Clercq (6) and Smith et al. (17) demonstrated the importance of the nature of the host cells in quantitation of antiviral drug potency. For example, they found that the order of decreasing drug potency changed from $(E)$-5-(2-bromovinyl)-2' ${ }^{\prime}$-deoxyuridine $>$ FIAC $>$ ACV to FIAC $>\mathrm{ACV}>(E)$-5-(2-bromovinyl)-2'-deoxyuridine depending upon which cell system was employed. Another consideration for in vitro testing of antiviral agents concerns the question of how many different virus strains should be tested when comparing them. Some investigators include several strains, whereas others prefer a representative strain of HSV-1, such as KOS or McKrae, which has been well characterized in many different laboratories $(6,17)$. For our in vitro antiviral drug tests, we employed wellcharacterized herpesvirus strains and SRK and Vero cells as host cell systems primarily on the basis of availability and susceptibility to HSV infections. We also had some variability when testing antiviral agents against four different HSV strains in these cell systems. However, FMAU and ACV still displayed the most potent antiviral activity against HSV-1 and HSV-2 in both host cell systems.

The cytotoxicity of FMAU was reported previously to be minimal, as determined by trypan blue dye exclusion with normal Vero, WI-38, and NC-37 cell proliferation (13). Minimal cytotoxicity of FMAU was confirmed by Fox et al. in Vero and human fibroblast cells (10) and now by this laboratory in Vero and SRK cells.

Our studies with the rabbit ocular model indicate that FMAU has useful antiviral potential against herpesvirus infection. The attempt that we made to determine the optimum FMAU drug concentration for topical use was limited by the amount of experimental drug available when the study was performed. It is possible that a concentration greater than the $0.2 \%$ FMAU tested might prove to be even better since topical FMAU is well tolerated by the rabbit eye. Chemotherapy was initiated at a time when the experimental ocular HSV-1 infection first became obvious to the examiner (day 4 postinoculation). By this time, however, the virus has already gained entry into the nervous system. We have found (unpublished data) that HSV-1 can enter the trigeminal ganglia within 24 $h$ after contact with intact corneal epithelial cells. Virus isolation from sacrificed animals confirmed that HSV-1 had colonized one or both of the trigeminal ganglia of most rabbits, irrespective of their treatment regimen. It is probably not possible to eradicate the latent virus from the nervous system with currently available chemotherapeutic agents (9).

As reported previously, drug-resistant strains of HSV have been associated with most antiviral drugs $(1,19)$. Two distinct loci in HSV-1 have been reported to confer resistance to $\operatorname{ACV}(3)$. Early clinical use of ACV has resulted in at least two reports of ACV resistance $(4,5)$. In the case of FMAU, it is premature to predict whether drug resistance will be a problem. However, our initial attempts to isolate FMAU-resistant HSV from rabbits have been unsuccessful. Several HSV isolates taken from rabbit tear film and tissue samples after topical FMAU therapy did not demonstrate any enhanced drug resistance when studied by plaque reduction testing (data not shown). However, failure to isolate FMAUresistant HSV from tear films and nervous system tissue samples does not mean that the development of increased resistance is not a possibility. We are continuously checking virus isolates for increased resistance.

\section{ACKNOWLEDGMENTS}

This work was supported in part by Public Health Service grants EY00858, EY02957, EY03040, CA-18601, and CA18856 from the National Institutes of Health, and by the Discovery Fund.

\section{LITERATURE CITED}

1. Bauer, D. J. 1977. Viruses in relation to chemotherapy, p. 16. In The specific treatment of virus diseases. University Park Press, Baltimore.

2. Brigden, D., A. E. Rosling, and T. Ravenscroft. 1981 Acyclovir-a review of the preclinical and early clinical data of a new antiherpes drug. Antiviral Res. 1:203-212.

3. Coen, D. M., and P. A. Schafter. 1980. Two distinct loci confer resistance to acycloguanosine in herpes simplex virus 1. Proc. Natl. Acad. Sci. U.S.A. 77:2265-2269.

4. Collins, P., and D. J. Bauer. 1977. Comparison of activity of herpes virus inhibitors. J. Antimicrob. Chemother. 3(Suppl. A):73-81.

5. Crumpacker, C. S., L. E. Schnipper, S. I. Marlowe, P. N. Kowalski, B. J. Hershey, and M. J. Levin. 1982. Resistance to antiviral drugs of herpes simplex virus isolated from a patient treated with acyclovir. N. Engl. J. Med. 306:343-345. 
6. De Clereq, E. 1982. Comparative efficacy of antiherpes drugs in different cell lines. Antimicrob. Agents Chemother. 21:661-663.

7. Elion, G. B., P. A. Furman, J. A. Fyfe, P. deMiranda, L. Beauchamp, and H. J. Schaeffer. 1977. Selectivity of action of an antiherpetic agent, 9-(2-hydroxyethoxymethyl) guanine. Proc. Natl. Acad. Sci. U.S.A. 74:5716-5720.

8. Field, H. J., S. E. Bell, G. B. Elion, A. A. Nash, and P. Wildy. 1979. Effect of acycloguanosine treatment on acute and latent herpes simplex infections in mice. Antimicrob. Agents Chemother. 15:554-561.

9. Field, H. J., and P. Wildy. 1981. Recurrent herpes simplex: the outlook for systemic antiviral agents. Br. Med. J. 282:1821-1822.

10. Fox, J. J., C. Lopez, and K. A. Watanabe. 1980. 2'Fluoro-arabinosyl pyrimidine nucleoside: chemistry, antiviral, and potential anticancer activities, p. 27-40. In F. G. De Las Heras and S. Vegas (ed.), Proceedings of the VIIth International Symposium on Medicinal Chemistry. Pergamon Press, Elmsford, N.Y.

11. Kaufman, H. E. 1978. Herpetic keratitis. Invest. Ophthalmol. Visual Sci. 17:941-957.

12. Kwon, B. S., L. P. Gangarosa, K. D. Burch, J. deBack, and J. M. Hill. 1981. Induction of ocular herpes simplex virus shedding by iontophoresis of epinephrine into rabbit cornea. Invest. Ophthalmol. Visual Sci. 21:442-449.

13. Lopez, C., K. A. Wanatabe, and J. J. Fox. 1980. 2'Fluoro-5-iodo-aracytosine, a potent and selective antiherpesvirus agent. Antimicrob. Agents Chemother. 17:803-806.

14. Nesburn, A. B., E. C. Dunkel, and M. D. Trousdale. 1980.
Enhanced HSV recovery from neuronal tissues of latently infected rabbit. Proc. Soc. Exp. Biol. Med. 163:398-401.

15. Pavan-Langston, D. R. 1979. Ocular viral diseases, p. 253-303. In G. J. Galasso, T. C. Merigan, and R. A. Buchanan (ed.), Antiviral agents and viral diseases of man. Raven Press, New York.

16. Sibrack, C. D., L. T. Gutman, C. M. Wilfert, C. McLaren, and D. Barry. 1981. Altered pathogenicity of acyclovir resistant HSV from an immune deficient child. Pediatr. Res. 15:621.

17. Smith, K. O., K. S. Galloway, W. L. Kennell, K. K. Olgivie, and B. K. Radatus. 1982. A new nucleoside analog, 9:[2-hydroxy-1-(hydroxymethyl)ethoxyl]methyl]guanine, highly active in vitro against herpes simplex virus types 1 and 2. Antimicrob. Agents Chemother. 22:55-61.

18. Trousdale, M. D., A. B. Nesburn, and C. A. Miller. 1981. Assessment of acyclovir on acute ocular infection induced by drug-resistant strains of HSV-1. Invest. Ophthalmol. Visual Sci. 20:230-235.

19. Trousdale, M. D., A. B. Nesburn, K. A. Watanabe, and J. J. Fox. 1981. Evaluation of the antiherpetic activity of 2'-fluoro-5-iodo-ara-C in rabbit eyes and cell cultures. Invest. Ophthalmol. Visual Sci. 21:826-832.

20. Watanabe, K. A., U. Reichman, K. Hirota, C. Lopez, and J. J. Fox. 1979. Nucleosides. 110. Synthesis and antiherpes virus activity of some 2 '-fluoro-2'-deoxyarabinofuranosylpyrimidine nucleosides. J. Med. Chem. 22:2124.

21. Weiner, B. J. 1971. Statistical principles in experimental design, 2nd ed. McGraw-Hill Book Co., New York. 